\title{
Rotational Excitation of Diatomic Molecule: Time Dependent Study
}

\author{
A. MAAN ${ }^{a, b *}$, A. TYAGi ${ }^{c}$ AND V. PRASAD ${ }^{c}$ \\ ${ }^{a}$ Department of Physics, Chaudhary Devi Lal University, Sirsa-125055, Haryana, India \\ ${ }^{b}$ Department of Physics, Pt.N.R.S.G.C.Rohtak, Maharshi Dayanand University, \\ Rohtak-124001, Haryana, India (On Leave) \\ ${ }^{c}$ Department of Physics, Swami Shradhanand College, University of Delhi, Delhi, Delhi-110036, India
}

(Received July 20, 2017; in final form January 13, 2018)

\begin{abstract}
Recent experimental evidences have prompted us to study the dynamics of laser-assisted rotational excitation of a diatomic molecule due to an ion impact. The collision time between the ion and the molecule is very small (few atomic units) and the laser pulses considered are of picosecond range. We study the evolution of the rotational probabilities by varying the various laser parameters (pulse shape, field strength, pulse width, frequency) and collision parameters (impact parameter $b$, collisional velocity $v$ ). It is found that the probabilities of the rotational states depend strongly on the laser pulse parameters and the collision parameters. The study is more emphasised by studying $\left\langle J^{2}\right\rangle$ (a parameter defining the extent of the rotational excitation) and $\left\langle J_{z}\right\rangle$ for the system under concern. For higher value of $b$, the maximum value obtained by $\left\langle J^{2}\right\rangle$ increases and vice versa. Also, the molecule enters the transient mode for large $b$ and $v$.
\end{abstract}

DOI: 10.12693/APhysPolA.133.1266

PACS/topics: collision, rotational excitation, probabilities, impact parameter, collisional velocity

\section{Introduction}

The kinetics of low-temperature hydrogen plasmas (e, $\mathrm{H}, \mathrm{H}^{+}, \mathrm{H}^{-}, \mathrm{H}_{2}, \mathrm{H}_{2}^{+}, \mathrm{H}_{3}^{+}$) existing in various astrophysical and laboratory environments is quite complex. This is due to the fact that the number of quantum states of atomic and molecular species involved in the collision kinetics is very large (i.e. electronic and ro-vibrational excited states) and also the atomic and molecular collision kinetics are coupled by many interconversion processes. The information regarding the characteristics like reaction cross-sections or rate coefficients provides the key for interpretation of the observed properties of the atomic and molecular species considered.

It is well acquainted that the spectrum of a diatomic molecule changes significantly in the presence of an intense laser field which shows important non-linear effects during collisions in the presence of the laser field. The laser-induced collisions leading to energy transfer is an optical process where the laser field is applied during the collision between the two particles which could be the combination of ion with atoms or molecules [1-8], atom with atom [9-11] etc. The laser-induced collision was proposed in the 1970s $[12,13]$. These processes find considerable importance in the field of laser-induced chemistry [14], development of powerful lasers etc. and have been investigated both theoretically [15-20] and experimentally [5, 6, 21-23].

*corresponding author; e-mail: anjalimaan2607@gmail.com
The inelastic scattering due to vibration and rotation is one of the simplest in the energy transfer processes and finds significant importance for understanding the kinetics of gas phase chemical reactions, energy balance in plasma, etc. The energy transfer collisions also influence the rate of radiation and thus determine the population and depopulation of the excited states. Many theoretical and experimental studies have been performed to study the ion-impact excitation of a diatomic molecule in the absence of the laser field [24]. The advent of laser has made it important to understand the energy transfer process in molecular collisions in the presence of radiation field. The exchange of the quanta of energy during the process of collision plays a significant role in the mechanism of lasers.

The laser-induced collisional energy transfer i.e. an optical process where a laser field is applied during the process of collision between the two different particles in order to induce energy transfer from a level (of one collisional species) to other level (of another collisional species), has been studied thoroughly [9-11]. The said process cannot occur unless two mechanisms viz. collision and radiative interactions occur. Neither of the two interactions can singly induce interparticle transition. The reaction representing laser-induced energytransfer process (between two atoms A1 and A2) can be expressed as

$$
\mathrm{A} 1^{*}(\mathrm{i})+\mathrm{A} 2\left(\mathrm{i}^{\prime}\right)+\hbar \omega \rightarrow \mathrm{A} 1(\mathrm{f})+\mathrm{A} 2^{* *}\left(\mathrm{f}^{\prime}\right) .
$$

During the process of collision between two different atoms, a strong dressing laser field is used to drive the ac stark splitting of the intermediate and the final state while the weak inducing laser field causes the interparticle transition, thereby resulting in the modification of the 
excitation profile. The dressing laser field cannot only be utilised to study the ac Stark effect of atomic levels but can also be used to realize the efficient population transfer, which makes this theoretical system a promising one. The experimental evidence of the collisional energy transfer along with its dynamics has been explained in detail in a series of papers $[9,11]$. The interference effects due to the collision between the molecule and an ion has been observed [25, 26]. Also, the energy and angular distributions of electron emission from diatomic molecule by an ion impact has also been reported [27].

The collision processes can occur in a variety of ways, out of which few are as follows:

(A) Electron-impact processes:

The inelastic electron impact processes of an atom $\mathrm{A}$, in a quantum state characterised by principal and angular momentum quantum numbers $n$ and $l$, include

$$
\begin{aligned}
& \mathrm{e}+\mathrm{A}(n l) \rightarrow \mathrm{e}+\mathrm{A}\left(n^{\prime} l^{\prime}\right), \quad n^{\prime} \neq n \geq 1 \quad \text { (excitation), } \\
& \mathrm{e}+\mathrm{A}(n l) \rightarrow \mathrm{e}+\mathrm{A}^{+}+\mathrm{e}, \quad n \geq 1 \quad \text { (ionization), } \\
& \quad \mathrm{e}+\mathrm{A}(n l) \rightarrow \mathrm{A}^{-}+h \nu, \quad n \geq 1 \\
& \quad \text { (radiative electron attachment). }
\end{aligned}
$$

(B) Proton-impact processes:

The inelastic proton impact processes of an atom $\mathrm{A}$, in a quantum state characterised by principal and angular momentum quantum numbers $n$ and $l$, include

$$
\begin{aligned}
& \mathrm{H}^{+}+\mathrm{A}(n l) \rightarrow \mathrm{H}^{+}+\mathrm{A}\left(n^{\prime} l^{\prime}\right), \quad n^{\prime}>n, \\
& \mathrm{H}^{+}+\mathrm{A}(n l) \rightarrow \mathrm{H}^{+}+\mathrm{A}^{+}+\mathrm{e}, \quad n \geq 1, \\
& \mathrm{H}^{+}+\mathrm{A}(n l) \rightarrow \mathrm{H}^{+}+\mathrm{A}\left(n^{\prime} l^{\prime}\right), \quad n^{\prime} \approx n .
\end{aligned}
$$

(C) Atom-atom collision processes:

The inelastic collision of an atom $\mathrm{A}$, in a quantum state characterized by principal quantum number $n$, with the same atom in state $m$ (one of the atoms is in excited state) includes:

$$
\begin{aligned}
& \mathrm{A}(m)+\mathrm{A}(n) \rightarrow \mathrm{A}(m)+\mathrm{A}(n), \\
& n \neq m, \quad n, m \geq 1, \\
& \mathrm{~A}(m)+\mathrm{A}(n) \rightarrow \mathrm{A}^{+}+\mathrm{A}\left(\mathrm{n}^{\prime}\right)+\mathrm{e}, \\
& m, n \geq 2, \quad n^{\prime}<n, \quad \Delta E_{m n^{\prime}}>I_{n}
\end{aligned}
$$

The rotational excitation in molecules have been studied in scarce $[28,29]$. In the present work, we have developed the theory of collision aided rotational excitation of $\mathrm{HBr}$ molecule due to ion (proton) impact, in its ground electronic and vibrational state in the presence of a linearly polarized laser field. We assume that the collision time is quite small (is of the order of few atomic units) and the laser pulse is in picoseconds. We present an account of the general theory of the related process along with the basic equations involved and then discuss the dynamics of the system. The reaction of the laser-assisted collision of the present system can be expressed as

$\mathrm{H}^{+}+\operatorname{HBr}(v, J=0)+n \hbar \omega \rightarrow \mathrm{H}^{+}+\operatorname{HBr}\left(v, J=J^{\prime}\right),(10)$ where $v$ and $J$ corresponds to the vibrational and rotational quantum number, respectively. To solve the present problem, we make use of the fourth-order RungeKutta method. At present to our knowledge, no experimental and theoretical work is available to compare with the present study. However, it is expected that with the advances in the laser technology, such experiments on ion-molecule systems be performed in near future. Further, we show the effect of collision process on the rotational excitation and on the extent of rotational excitation (defined by a parameter explained in theory) with respect to various collision parameters. The time evolution of collision process in laser field in future will help in developing techniques for molecular imaging.

The structure of the paper is organized as follows. In Sect. 2, we present the theoretical approach used in our calculations, followed by Sect. 3, which present our results and the respective discussions and finally in Sect. 4, the conclusions are drawn.

\section{Theoretical approach}

We consider the collision between an ion and a polar diatomic molecule in the presence of linearly polarised laser pulse. We have taken here the example of $\mathrm{HBr}$ molecule which is one of the most studied polar molecules. Figure 1 represents the co-ordinate system formed by ion (proton) and the molecule in the center of mass frame, where A represents the ion(proton) $\left(\mathrm{H}^{+}\right)$and $\mathrm{B}$ - the centre of mass of the $\mathrm{HBr}$ molecule. The quantities $b$ and $v$ represents the impact parameter and collisional velocity respectively.

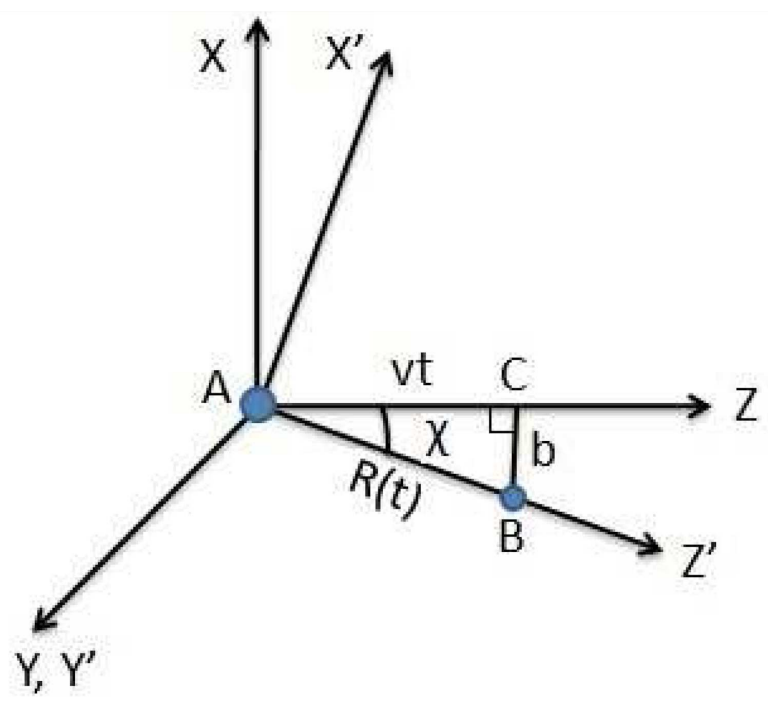

Fig. 1. Co-ordinate system formed by an ion (proton) and the molecule. 
We assume the laser field to be

$$
\boldsymbol{E}(t)=\hat{\epsilon} \boldsymbol{E}_{0}(t) \cos (\omega t)
$$

of amplitude $E_{0}(t)$ and the optical frequency $\omega$. We have $\hat{\epsilon}$ as the direction of polarization of the laser field and $E_{0}(t)=E_{0} p(t)$, where $E_{0}$ is the field strength and $p(t)$ is the pulse envelope for a half-cycle pulse (HCP) or a square pulse (SQP) defined as:

$$
\begin{aligned}
& p(t)=\sin ^{2}\left(\pi t / t_{p}\right), \quad 0<t<t_{p} \quad, \mathrm{HCP}, \\
& p(t)=1, \quad \text { for SQP }
\end{aligned}
$$

where $t_{p}$ is pulse duration. The Schrödinger equation, for this model, is (in atomic units)

$\iota \frac{\partial \psi}{\partial t}(\boldsymbol{r}, t)=\left[H_{0}(\boldsymbol{r})+V_{C}(\boldsymbol{r}, \boldsymbol{R}, \gamma)+V_{L}(\boldsymbol{E}, \omega)\right] \psi(\boldsymbol{r}, t)$,

where the first term on the right hand side represents the unperturbed Hamiltonian of the molecule and the second term is the interaction potential between the ion and the molecule and is given by [4]:

$$
\begin{aligned}
& V_{C}(\boldsymbol{r}, \boldsymbol{R}, \gamma)=-\frac{\mu(\boldsymbol{r}) \cdot \boldsymbol{R}}{R^{3}}=-\frac{\mu(\boldsymbol{r}) \boldsymbol{R} \cos \chi}{R^{3}} \\
& =-\frac{\mu(\boldsymbol{r}) v t}{\left(b^{2}+v^{2} t^{2}\right)^{3 / 2}}
\end{aligned}
$$

where $\mu(\boldsymbol{r})$ is the dipole moment operator of the molecule, $\cos \chi=\frac{v t}{R}$ and $\boldsymbol{R}$ is defined as:

$$
\boldsymbol{R}(t)=b+v t, \quad b \cdot v=0
$$

Here $\boldsymbol{R}(t)$ is the position vector of the ion (proton) with respect to the centre of mass of the molecule, $v$ is the collisional velocity and $b$ is the impact parameter. Also, $\gamma$ is the angle between $\boldsymbol{r}$ and $\boldsymbol{R}$. Figure 2 shows how the asymmetric collision pulse changes with the changes in the collisional velocity $(v)$ and the impact parameter $(b)$. The term $V_{L}(\boldsymbol{E}, \omega)$ is the laser molecule interaction, i.e.

$$
V_{L}(\boldsymbol{E}, \omega)=-\mu(\boldsymbol{r}) \cdot E(t)=-\mu(\boldsymbol{r}) E(t) \cos \theta,
$$

where $\theta$ is the angle between the polarisation vector of the laser and the body fixed axis. Both the collisional and the laser pulse are applied at time $t=0$, i.e.
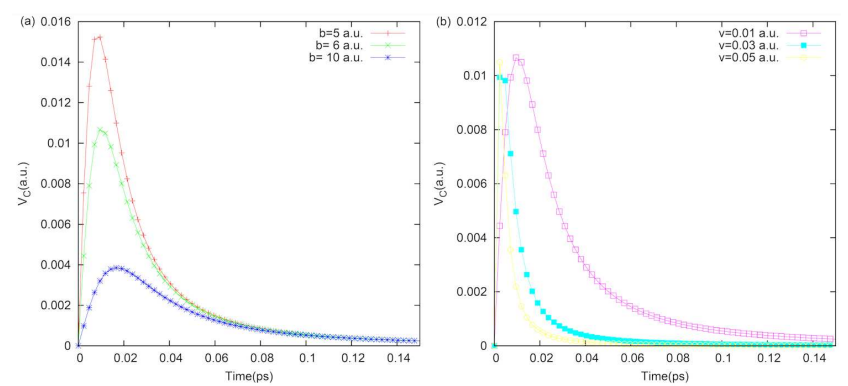

Fig. 2. $V_{C}$ (a.u.) as a function of time (in ps) for three different values of impact parameter $b$ (a) with $v=0.01$ a.u. and for three different values of collisional velocity $v$ (b) with $b=6$ a.u. The denotation of the curves is shown in parts $(a, b)$. there is no delay time between the two pulses. We have solved Eq. (14) using fourth-order Runge-Kutta method [30-32] with the initial condition taken as molecule being in the ground rotational $J=M=0$ state at $t=0$ state. This method is quite suitable in obtaining rotational probabilities as their is no dressing by the continuous laser field (which require alternate methods like quasi-energy technique as used by other groups [2-4]). In fact, we have applied a laser pulse of short duration. The parameter $\left\langle J^{2}\right\rangle$ defines the extent of the rotational excitation and is defined as follows [33]:

$$
\left\langle J^{2}\right\rangle=\sum_{J}\left|C_{J}\right|^{2} J(J+1) .
$$

Also,

$$
\left\langle J_{Z}\right\rangle=\sum_{J}\langle J \cos \theta\rangle=\sum_{J} J\langle\cos \theta\rangle .
$$

\section{Results and discussion}

In the present paper, we have investigated the results that illustrate the qualitative features of rotational transitions of a polar diatomic molecule $(\mathrm{HBr})$ due to an ion (proton) impact in the presence of linearly polarised electromagnetic radiation. $\mathrm{HBr}$ is a polar molecule with moderate permanent dipole moment $\mu=0.82876 \mathrm{D}$ and rotational constant $B=8.348296 \mathrm{~cm}^{-1}[31,32]$. The rotational constant of $\mathrm{HBr}$ shows that the rotational period of the molecule is approximately $2.04 \mathrm{ps}$ [34]. We assume that the collision time $\left(t_{c}\right)$ is quite short and also the laser pulse $\left(t_{p}\right)$ is of short duration i.e. $0.2 \mathrm{ps}$ (unless otherwise indicated). The frequency is taken to be zero (unless otherwise indicated). The solution of the Schrödinger equation is obtained using fourth-order Runge-Kutta method as described in Sect. 2. In our calculations we have taken the lowest ten rotational levels $(J=0,1, \ldots ., 9)$ and the figures with no indicated probability of any particular rotational level show the zero probability of that rotational state. The laser pulse taken is HCP unless mentioned otherwise.

In Fig. 2 we present the time evolution of the interaction parameter $V_{C}$. Part (a) shows $V_{C}$ for three different values of the impact parameter $(b)$ while part (b) shows the same for three different values of the collisional velocity $(v)$. We find that an increase in the impact parameter reduces the sharpness in the peak of the asymmetric pulse thereby reducing the force being applied in the form of "kick" to the target molecule. Also, the decrease in the collisional velocity $(v)$ increases the area of the collision pulse, thereby increasing its energy. Thus, by proper choice of the parameters of the collision pulse, we can modify the evolution of the rotational probabilities of the system under concern.

Figure 3 depicts the time-evolution of the probabilities of various rotational states. The probabilities of the levels zero to nine (i.e. ten levels) have been shown in two separate figures. The values of the field intensity $E_{0}$, col- 

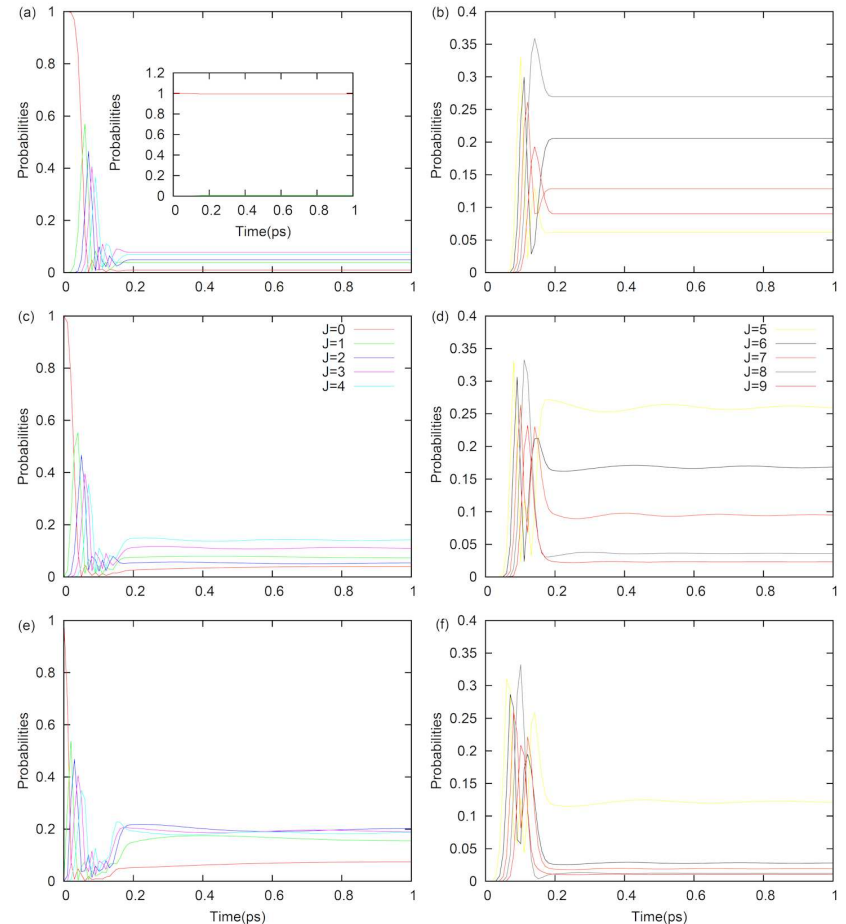

Fig. 3. Rotational probabilities as a function of the time (ps). Here, pulse duration $t_{p}$ is $0.2 \mathrm{ps,} \mathrm{colli-}$ sional velocity $v$ is 0.01 a.u., and the field strength $E_{0}=10^{-2}$ a.u. The impact parameter $b$ is 10 a.u. (in parts $(\mathrm{c}, \mathrm{d})), 6$ a.u. (in parts $(\mathrm{e}, \mathrm{f})$ ), and $V_{C}=0$ (in parts $(a, b))$. The laser pulse applied is a HCP. The inset in Fig. 3a shows the effect of low field strength i.e. $E_{0}=10^{-4}$ a.u. on the rotational probabilities. The denotation of curves is shown in Fig. 3c,d.

lisional velocity $v$ and the pulse duration $t_{p}$ are the same in all the figures and are $10^{-2}, 0.01$, and $0.2 \mathrm{ps}$, respectively. The only difference is in the value of the impact parameter $b$ which is 10 a.u. in Fig. 3c and $d$ and 6 a.u. in Fig. 3e and f. However, in Fig. 3a and b, there is no collision (i.e. only the $V_{L}$ is taken into consideration). For a particular value of $E_{0}, v, t_{p}$ and $b$ (say 10 a.u.), the transition probabilities of the states $(J=0, \ldots, 4)$ are shown in Fig. 3c whereas for the levels $(J=5, \ldots, 9)$ it is shown in Fig. 3d. The figure clearly depicts that for lower value of impact parameter (in Fig. 3e,f) i.e. when the projectile is near the center of mass of the molecule, the probabilities of higher rotational states do not attain a higher value in spite of the large value of the field strength. On the other hand, if the value of the impact parameter $b$ is increased from 6 a.u. to 10 a.u., the higher rotational states get excited for the same value of $E_{0}$. This is because of the fact that, on increase in the impact parameter, the effect of the ion on the molecule becomes less influential and that of the field becomes more prominent. Thus, we can say that the impact parameter plays a significant role in the rotational excitation dynamics of the molecule. In addition to this, we also find that in the absence of the collision term, or in the presence of only the laser term, the excitation to higher rotational state increase. As can be seen in Fig. 3b and d, there occurs higher excitation to level $J=6,8$, whereas in Fig. 3d it occurs for levels $J=5,6$. This means that the collision term which is an asymmetric pulse is a distracting factor in causing rotational excitation to higher states for higher fields. However, at lower fields (say $E_{0}=10^{-4}$ a.u.) the rotational excitation is limited to ground state only (shown in inset of Fig. 3a). In addition to the above, we can say that as we decrease the sharpness of the peak of the asymmetric pulse i.e. from Fig. 3f to Fig. 3d and finally to Fig. 3b (where $V_{C}=0$ i.e. no peak at all), the probability of transition to higher rotational states becomes large.

Figure 4 shows the variation in the rotational probabilities as a function of time in picoseconds for the case when the laser pulse is HCP. The field strength, collisional velocity, and the impact parameter are taken to be $10^{-2}$, 0.01 , and 6 a.u., respectively. Figure $4 \mathrm{a}$,b shows the evolution of the rotational probabilities when both the laser pulse $V_{L}$ and the collision pulse $V_{C}$ are taken into consideration while Fig. 4c,d are meant for only the laser pulse. Also, Fig. 4e,f reflects the response of only the collision pulse. The figure clearly depicts that when both the laser and the collision pulse are applied (in Fig. 4a,b), the transition probabilities stay in lower rotational states, while they persist in higher rotational states for only the laser pulse. In case of only the collision pulse, the transition
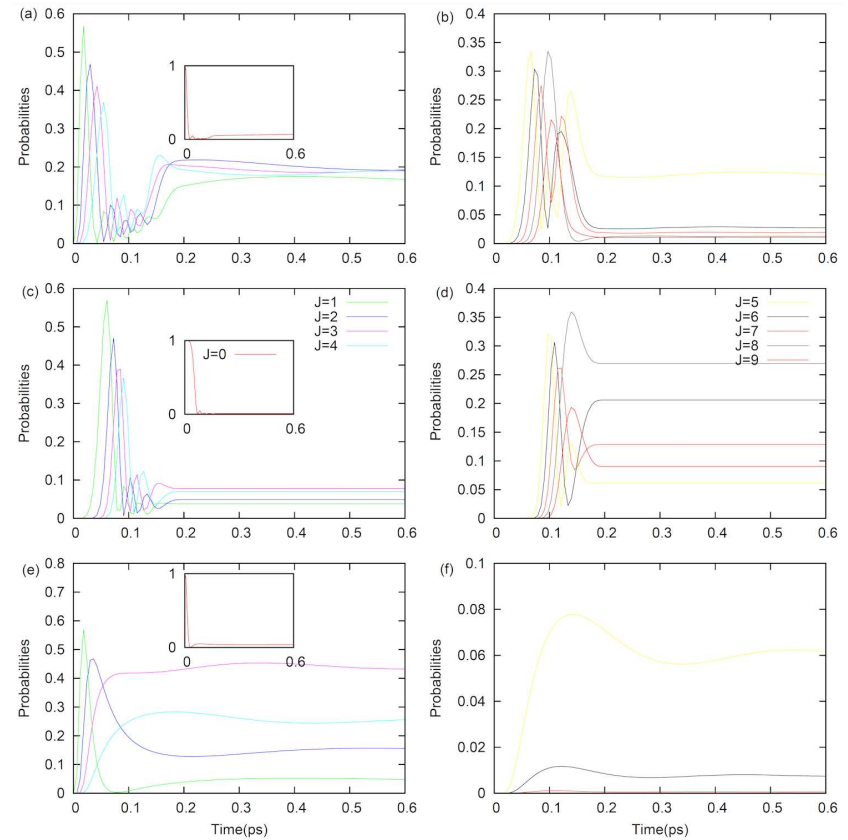

Fig. 4. Rotational probabilities as a function of the time (ps) for HCP as laser pulse. Here, pulse duration $t_{p}$ is $0.2 \mathrm{ps}$, collisional velocity $v$ is 0.01 a.u., the field strength $E_{0}=10^{-2}$ a.u. and the impact parameter $b$ is 6 a.u.. Figure 4a,b shows the effect of both the laser and the collision pulse while Fig. 4c,d presents the behaviour of only the laser pulse. Also, Fig. 4e,f is meant for only the collision pulse. The insets in Fig. 4a,c,e show the probabilities of $J=0$ state. The denotation of curves is shown in Fig. 4c,d. 
probability stays in lower rotational states with difference in their values. Actually, the only collision pulse is not sufficient to cause the rotational excitation to higher states. On the other hand, a single HCP laser pulse of strong field strength provides "kick" to molecule and causes transition in the probabilities to higher rotational states. A combination of both HCP and asymmetric collision pulse produces a net asymmetric pulse which also causes rotational excitation to lower rotational states.

Figure 5 is the same as Fig. 4 but for a square pulse. The figure shows that a combination of a square and a asymmetric collision pulse causes rotational excitation to higher states which was restricted to lower rotational states in case of HCP. However, the only square pulse of the said field strength causes the rotational excitation to intermediate rotational levels from $J=0$ to $J=1,2,3,4$ etc. The effect of only collision term is the same as in Fig. 4. Thus, we can say that a proper choice of the laser pulse shape can provide a way to decide the desired rotational excitation.
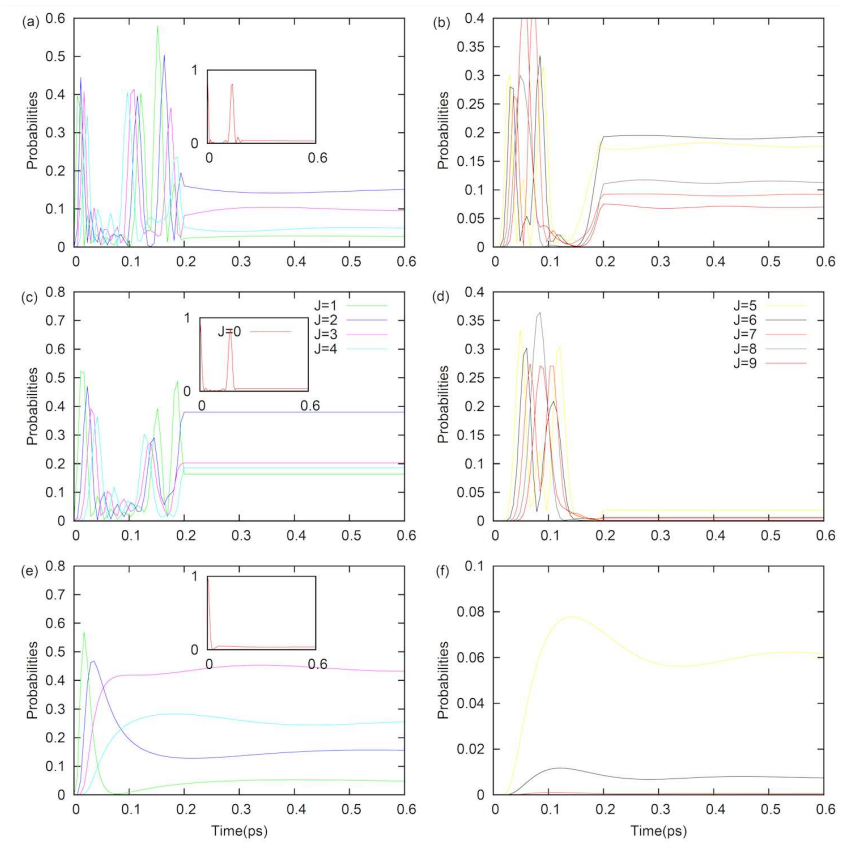

Fig. 5. The same as Fig. 4 but for SQP as laser pulse.

Figure 6 represents the variation in the transition probabilities as a function of impact parameter $b$. Figure $6 a$ and $\mathrm{b}$ shows the said variation for lower value of $E_{0}$ i.e. $10^{-6}$ a.u. whereas Fig. $6 \mathrm{c}$ and $\mathrm{d}$ for higher value of $E_{0}$ i.e. $10^{-2}$ a.u.. The collisional velocity and the pulse durations are the same for all the figures i.e. 0.01 a.u. and $0.2 \mathrm{ps}$, respectively. Due to the difference in the probabilities and also because of the large number of levels, the transition probabilities have been represented in two figures. The figure clearly depicts that the transition probabilities take the peak values and settle down to zero at lower value of impact parameter for lower value of the field strength. This is because of the fact that the higher
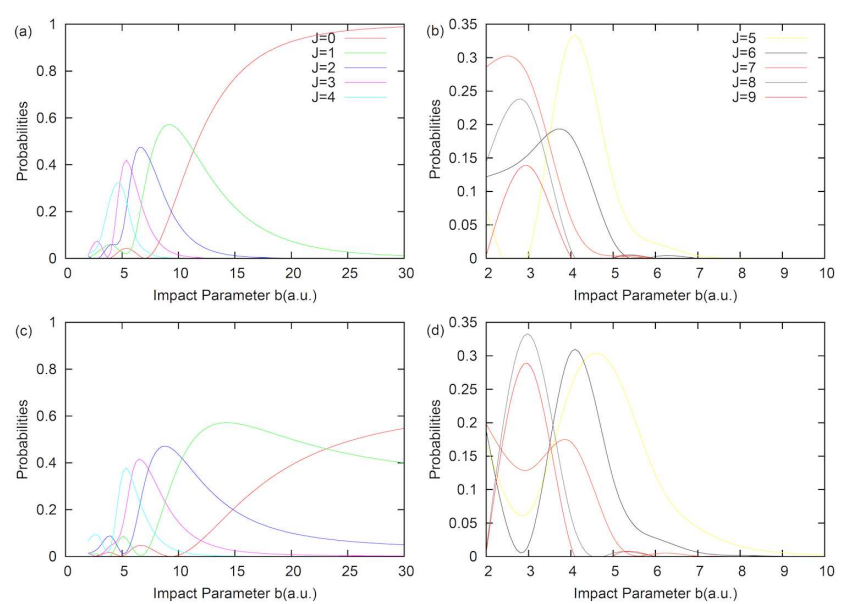

Fig. 6. Rotational probabilities as a function of the impact parameter $b$ (in a.u.). Here, pulse duration $t_{p}$ is $0.2 \mathrm{ps}$ and the collisional velocity $v$ is $0.01 \mathrm{a} . \mathrm{u}$. The field strength $E_{0}=10^{-6}$ a.u. (in Fig. 6a,b) and $E_{0}=10^{-2}$ a.u. (in Fig. 6c,d). The laser pulse is HCP. The denotation of curves is shown in Fig. $(a, b)$.

fields maintains higher probabilities of each rotational state for any value of the impact parameter. Thus, we can deduce that the impact parameter decides the maximum probability for any rotational level for a particular value of field strength.

Figure 7 depicts the variation in the probabilities as a function of field intensity $E_{0}$ which is varied from $10^{-6}$ a.u. to $10^{-1}$ a.u. The electric field limits are taken such as to avoid ionization of the molecule due to intense laser fields. The values of collisional velocity $v$ and the pulse duration $t_{p}$, are 0.01 a.u. and $0.2 \mathrm{ps}$, respectively, however, the impact parameter $b$ is 10 a.u. (in Fig. 7a
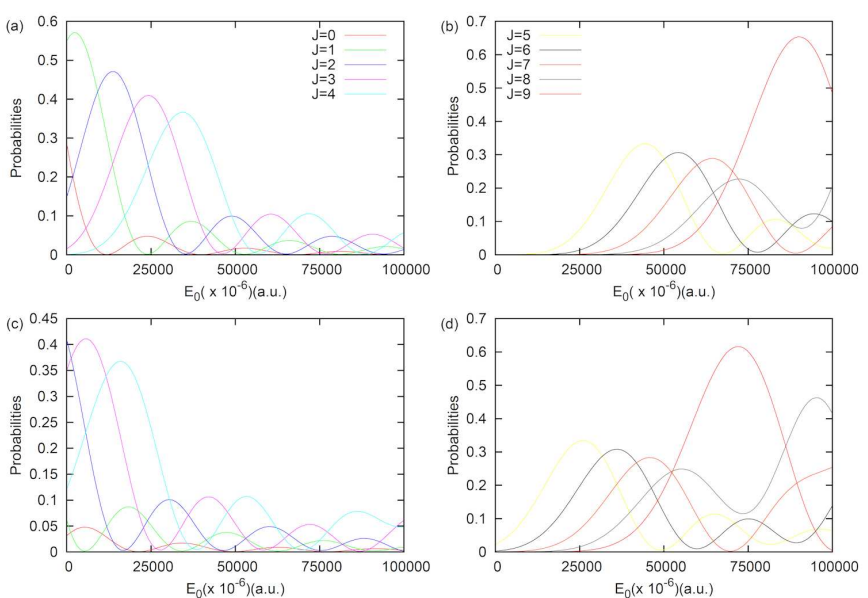

Fig. 7. Rotational probabilities as a function of the $E_{0}$ in a.u. Here, pulse duration $t_{p}$ is $0.2 \mathrm{ps}$ and collisional velocity $v$ is 0.01 a.u. The impact parameter $b$ is 10 a.u. (in Fig. 7a,b) and 6 a.u. (in Fig. 7c,d). The laser pulse is HCP. The denotation of curves is shown in Fig. 7a,b. 
and b) and 6 a.u. (in Fig. 7c and d). The figures clearly depict that the higher rotational states get excited with the increase in the field intensity $E_{0}$, thereby decreasing the probabilities of the lower rotational states as has also been shown [32]. But, here the difference lies in the fact how these rotational excitations vary with the change in value of the impact parameter. We can easily judge from the figure that for the lower value of impact parameter $b$, the higher rotational states take peak values of probability earlier, whereas the same occur for $b=10$ a.u. later. This is because, at lower value of impact parameter $b$, the ion-molecule interaction assists in rotational excitation, so that the probabilities acquires the peak values for lower $E_{0}$. Thus, we can say that by properly choosing the value of the field strength $E_{0}$ (within the limits not to ionize molecule) and the impact parameter $b$, we can cause the rotational excitation of the required rotational state to its peak value.

Figure 8 depicts the variation in the probabilities as a function of the collisional velocity in atomic units. The change is being studied for two different values of the field amplitudes $E_{0}$ i.e. a lower value $10^{-6}$ a.u. and a higher value $10^{-2}$ a.u. The transition probability of ten rotational states for any particular $E_{0}$ are being reflected in two separate figures for example for $E_{0}=10^{-6}$ a.u., Fig. $8 \mathrm{a}$ and $\mathrm{b}$ shows the transition probabilities and Fig. $8 \mathrm{c}$ and $\mathrm{d}$ for $E_{0}=10^{-2}$ a.u. The impact parameter and the pulse duration are taken to be 5 a.u. and $0.2 \mathrm{ps}$, respectively. Figure $8 \mathrm{c}$ and d shows that the rotational excitation is initiated at lower collisional velocities and then the probabilities attains almost a constant value for higher values of collisional velocities. Thus, we can say that even at higher field strengths, the low collisional velocities are more suitable in rotational excitation
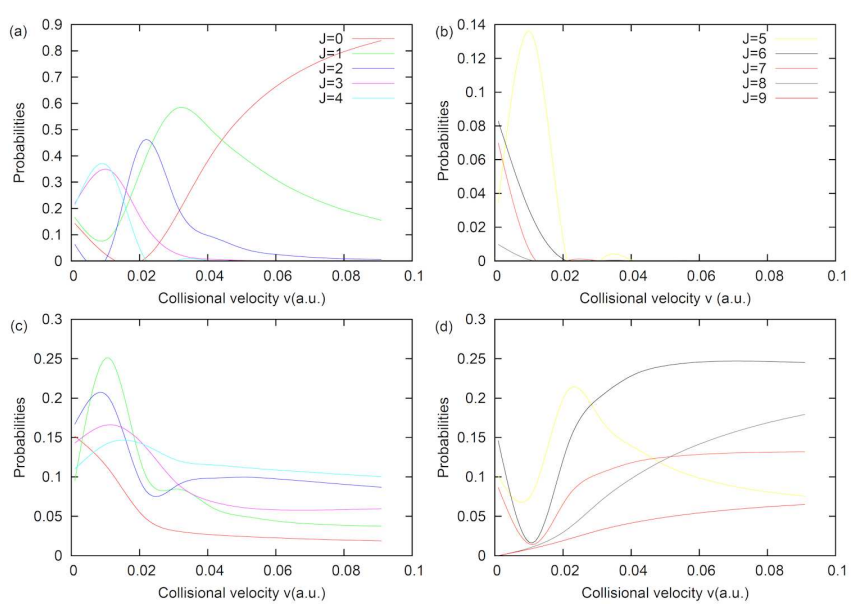

Fig. 8. Probability of various rotational states as a function of collisional velocity $v$ (in a.u.) for two different values of the field strength i.e. $E_{0}$ is $10^{-6}$ a.u. (in Fig. 8a,b) and $10^{-2}$ a.u. (in Fig. 8c,d). The impact parameter $b$ is 5 a.u. and the pulse duration $t_{p}=0.2 \mathrm{ps}$. The laser pulse is HCP. The denotation of the curves are shown in Fig. 8a,b. of almost all rotational states. Also, we can increase or decrease the probabilities of the rotational states by properly choosing the collisional velocity $v$ of the projectile for particular value of $E_{0}$. Figure $8 \mathrm{a}$ and $\mathrm{b}$ also reflects that for lower value of $E_{0}$ i.e. $10^{-6}$ a.u., the higher rotational states $(J=1, \ldots, 4)$ get excited for higher value of $v$ and finally the transition probabilities lowers to zero value for higher $v$. Also, for further higher rotational states (i.e. $J=5, \ldots, 9)$, the probability ends up for lower values of $v$. Thus, we can say that the probability does not sustain to higher values even on increasing the collisional velocity for lower value of $E_{0}$.

Figure 9 shows the variation in the probabilities with the change in frequency (in $\mathrm{cm}^{-1}$ ). Figure $9 \mathrm{a}$ and $\mathrm{b}$ shows the said variation with only the laser term $V_{L}$ only, whereas Fig. 9c and d shows the same for both $V_{L}$ and $V_{C}$. The field strength $E_{0}$, collisional velocity $v$, pulse duration $t_{p}$ and the impact parameter $b$ are assumed to be $10^{-3}$ a.u., 0.01 a.u., $0.2 \mathrm{ps}$, and 5 a.u., respectively. The oscillating behaviour in the probabilities is due to various resonances due to which the peaks are observable at different frequencies. However, these peaks tends to reduce on removing the collision term from the interaction terms. We also find that the oscillating behaviour in the probabilities is more probable for higher rotational states by including the collision term $V_{C}$. Thus, we can say that by including the collision term in the interaction Hamiltonian increases the number of resonances occurring at different frequencies.
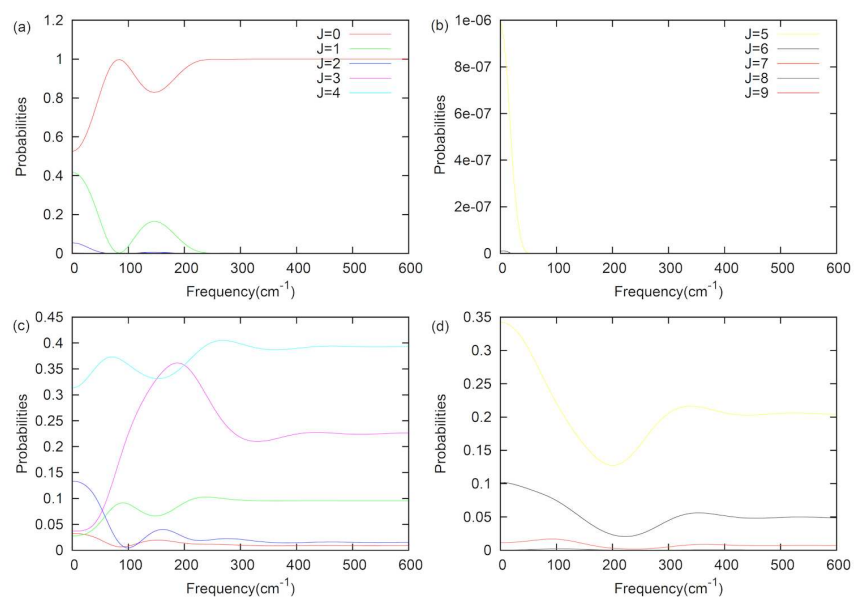

Fig. 9. Probability of various rotational states as a function of frequency (in $\mathrm{cm}^{-1}$ ) for with (in Fig. 9c,d) and without (in Fig. 9a,b) the collision term $V_{C}$. The impact parameter $b$ is 5 a.u. and the collisional velocity $v$ is 0.01 a.u. Also, $E_{0}=10^{-2}$ a.u. and $t_{p}=0.2$ ps. The laser pulse is HCP. The denotation of the curves is shown in Fig. 9a,b.

Figure 10 shows the variation in the probabilities with the change in the pulse duration $t_{p}$ (in ps). The collisional velocity $v$, impact parameter $b$ and the field strength $E_{0}$ are taken to be $0.01,5$, and $10^{-2}$ a.u., respectively. The behaviour of the probabilities with the 

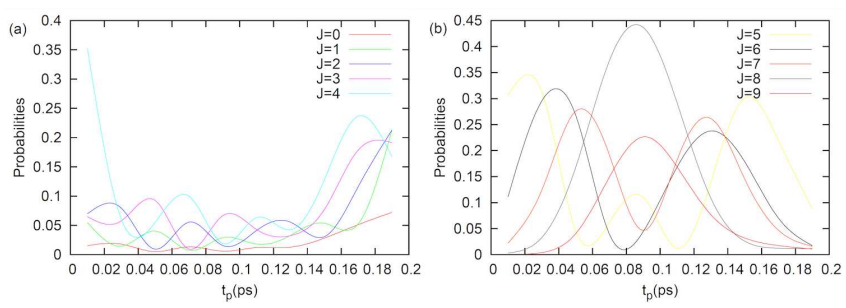

Fig. 10. Probability of various rotational states as a function of pulse duration $t_{p}$. The impact parameter $b$ is 5 a.u., the collisional velocity $v$ is 0.01 a.u. and $E_{0}=10^{-2}$ a.u. The laser pulse is HCP. The denotation of the curves is shown in Fig. 10a,b.

pulse duration is oscillatory in nature. It is found that the immediate excited states $(J=1, \ldots, 4)$ take peak values near the pulse duration $0.2 \mathrm{ps}$ for the $\mathrm{HBr}$ molecule. However, the excited states $(J=5, \ldots, 9)$ keep on oscillating for changes in the value of the pulse duration and take peak values at different values of $t_{p}$. This is because of the fact that, as the field is high, the higher rotational states get excited even for lower value of the pulse width. However, the lower rotational states get excited for the pulse duration in the non-adiabatic limit $\left(t_{p} \approx T_{\text {rot }} / 10\right)$. Thus, the pulse duration can prove to be an important criterion in manipulating the rotational excitation of the molecule.

Figure 11a depicts the time-evolution of $\left\langle J^{2}\right\rangle$ for three different values of impact parameter $b$. The pulse duration $t_{p}$, collisional velocity $v$ and the field strength $E_{0}$ are taken to be $0.2 \mathrm{ps}, 0.01$ a.u., and $10^{-2}$ a.u., respectively. The figure clearly depicts that $\left\langle J^{2}\right\rangle$ goes along with the pulse and then settles down to a constant value. Also, for a higher value of $b$ (impact parameter), we find that the constant value settles for larger value of $\left\langle J^{2}\right\rangle$. Thus, we can say that when the projectile is at a larger distance from the molecule i.e. when is under the effect of weak Coulomb potential, the extent of rotational excitation $\left(\left\langle J^{2}\right\rangle\right)$ increases. This implies that the possibility of transition to higher states is more probable.
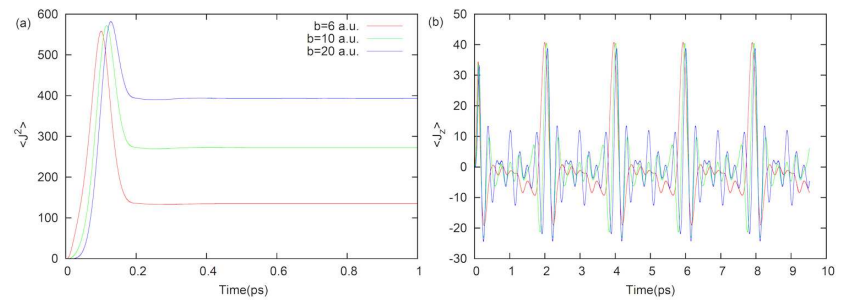

Fig. 11. Time-evolution of $\left\langle J^{2}\right\rangle$ and $\left\langle J_{z}\right\rangle$ for three different values of impact parameter $b$. The pulse duration $t_{p}$, collisional velocity $v$ and the field strength are $0.2 \mathrm{ps}$, 0.01 a.u., and $10^{-2}$ a.u., respectively. The laser pulse is HCP type. The denotation of the curves are in Fig. 11a.

At the same time, Fig. $11 \mathrm{~b}$ represents the timeevolution of $\left\langle J_{z}\right\rangle$ for the above said three different values of $b$. The values of $t_{p}, v$ and $E_{0}$ are the same as defined in Fig. 11a. The figure shows revivals for all the three cases of impact parameters, but we find that the maximum of $\left\langle J_{z}\right\rangle$ is higher for lower $b$ and decreases for higher $b$. Also, we find that between the primary revivals, there occur secondary and tertiary revivals and these decrease with increase in the value of $b$. It means that for larger value of the impact parameter, the molecule enters the transient mode.

Figure $12 \mathrm{a}$ is the same as Fig. 11a but for different values of collisional velocity $v$ keeping the value of pulse duration $t_{p}$, impact parameter $b$ and the field strength $E_{0}$ to be constant as $0.2 \mathrm{ps}, 20$ a.u., and $10^{-2}$ a.u., respectively. The three different values of collisional $v$ are taken to be $0.01,0.05$, and 0.1 a.u. Figure 12 a reflects higher rotational excitation (i.e. higher $\left\langle J^{2}\right\rangle$ ) for higher $v$. This is because a higher value of $v$ implies larger value of energy of the projectile leading to higher rotational excitation of the molecule.
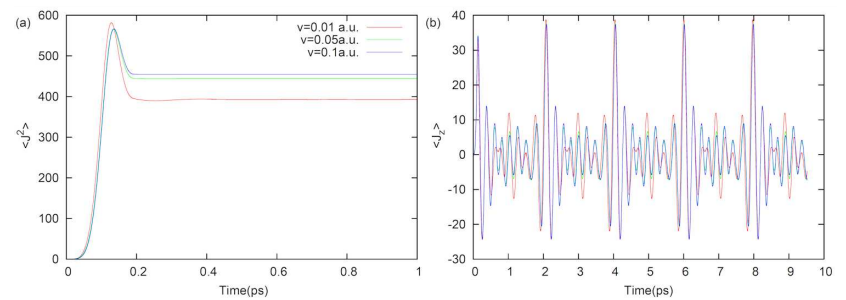

Fig. 12. Time-evolution of $\left\langle J^{2}\right\rangle$ and $\left\langle J_{z}\right\rangle$ for three different values of collisional velocity $v$. The pulse duration $t_{p}$, impact parameter $b$ and the field strength are $0.2 \mathrm{ps,}$ 20 a.u. and $10^{-2}$ a.u., respectively. The laser pulse is HCP type. The denotation of the curves are in Fig. 12a.

Also, Fig. 12b depicts time-evolution of $\left\langle J_{z}\right\rangle$ for three different values of $v$. The figure shows higher maxima of $\left\langle J_{z}\right\rangle$ for lower value of $v$. Here, also there occurs secondary and tertiary maxima between the primary maxima, but the oscillations increase for higher $v$ showing that the molecule is in transient mode for lower $v$.

\section{Summary and conclusion}

A theoretical investigation of the laser-assisted collisional rotational excitation between an ion and the molecule is reported for a linearly polarized laser radiation. The results shows that the transition probabilities depends strongly on the collision parameters like impact parameter $b$ and collisional velocity $v$. The various laser parameters like pulse duration $t_{p}$, pulse shape, frequency $\omega$ and the field strength $E_{0}$ also affects the dynamics. The extent of the rotational excitation $\left\langle J^{2}\right\rangle$ and $\left\langle J_{z}\right\rangle$ show strong dependence on the impact parameter $b$ and the collisional velocity $v$. Such collision processes finds great importance for the development of short-wavelength laser sources. Also, the ability to excite the special target level of the chosen particle permits potential applications of laser-induced collisional energy 
transfer (LICET) [9, 10, 35] in controlling pathways of chemical reactions, thus making a detailed understanding of atomic dynamical processes possible. In addition, in case of laser-assisted collisions due to ion-molecule interaction, the laser acts as an external control parameter for the reaction mechanism [2], for example by changing the ellipticity of the polarised laser field.

\section{Acknowledgments}

One of us (A.M.) acknowledges the help from University Grants Commission (UGC) for financial support under FIP scheme for teacher fellow.

\section{References}

[1] L.B. Madsen, J.P. Hansen, L. Kocbach, Phys. Rev. Lett. 89, 093202 (2002).

[2] V. Prasad, K. Yamashita, Chem. Phys. Lett. 426, 8 (2006).

[3] V. Prasad, B. Sharma, M. Mohan, Z. Phys. D 36, 47 (1996).

[4] M. Mohan, V. Prasad, J. Phys. B 24, L81 (1991).

[5] Z. Lu, Y. Sun, L. Ma, J. Liu, J. Phys. Chem. C 121, 9714 (2017)

[6] H. Zhang, Z. Lu, R. Fan, D. Chen, Laser Phys. 19, 947 (2009).

[7] A. Ortner, A. Franx, A. Blazeric, M. Roth, Phys. Rev. E 91, 023104 (2015).

[8] F.J.D. Gutierrez, R.C.-Trujillo, J. Phys. B 48, 135202 (2015).

[9] Z.Z. Lu, Y.L. Sun, L. Ma, J.F. Liu, J. Phys. Chem. A 119, 1957 (2015).

[10] Z.Z. Lu, D.Y. Chen, R.G. Fan, Y.Q. Xia, Phys. Rev. A 85, 063402 (2012).

[11] Z.Z. Lu, D.Y. Chen, Y.Q. Xia, R.W. Fan, H.Y. Zhang, Opt. Express 18, 21062 (2010).

[12] R.W. Falcone, R.W. Green, Phys. Rev. A 15, 1333 (1977).

[13] B. Cheron, H. Lemry, Opt. Commun. 42, 109 (1982).

[14] T.F. George, J. Phys. Chem. 86, 10 (1982).
[15] M. Mohan, P. Chand, J. Chem. Phys. 71, 2207 (1979).

[16] M. Mohan, K.F. Milfeld, R.E. Wyatt, Chem. Phys. Lett. 99, 411 (1983).

[17] M. Mohan, P. Chand, B. Sharma, Mol. Phys. 54, 959 (1985).

[18] G.D. Billing, Chem. Phys. Lett. 127, 269 (1986).

[19] R. Buffa, S. Cavalieri, R. Eramo, L. Fini, Phys. Rev. A 52, 1382 (1995).

[20] M. Matera, M. Mazzoni, R. Buffa, Phys. Rev. A 36, 1471 (1987).

[21] P.P. Dingles, J.F. Delpech, J. Weiner, Phys. Rev. Lett. 44, 1663 (1980).

[22] P. Hering, R.R. Brooks, R.F. Curl, R.S. Judson, R.S. Lowe, Phys. Rev. Lett. 44 687(1980).

[23] Y.P. Hsu, M. Kumura, R.E. Olson, Phys. Rev. A 31 , 576 (1985).

[24] R.D. Levine, R.B. Bernstein, T.L.Yuan, Molecular Reaction Dynamics and Chemical Reactivity, Oxford Univ. Press, Oxford 1987.

[25] L.Ph.H. Schmidt, S. Schössler, F. Afaneto, M. Schöffler, K.E. Steibing, H. Schmidt-Böking, R. Dörner, Phys. Rev. Lett. 101, 173202 (2008).

[26] C.A. Tachino, F. Martin, R.D. Rivarola, J. Phys. B 45, 025201 (2012).

[27] A. Mondal, C.R. Mandal, M. Purkail, Nucl. Instrum. Methods Phys. Res. B 353, 28 (2015).

[28] Y. Itikawa, N. Mason, Phys. Rep. 414, 1 (2005).

[29] A.S. Dickinson, D. Richards, J. Phys. B 8, 2846 (1975).

[30] A. Maan, D. Ahlawat, V. Prasad, Chem. Phys. Lett. 650, 29 (2016).

[31] A. Tyagi, U. Arya, B. Vidhani, V. Prasad, Spectrochim. Acta A 129, 193 (2014).

[32] A. Tyagi, A. Maan, D.S. Ahlawat, V. Prasad, Spectrochim. Acta A 173, 13 (2017).

[33] T. Seideman, E. Hamilton, Adv. At. Mol. Opt. Phys. 52, 289 (2000).

[34] D. Pinkham, T. Vogt, R.R. Jones, J. Chem. Phys. 129, 064307 (2008).

[35] Z. Lu, Y.L. Sun, L. Ma, J.F. Liu, Acta Phys. Pol. A 27, 1620 (2015). 